\title{
Bordism of regularly defective maps
}

\author{
Stefan Bechtluft-Sachs, Ulrich Riegel, Jörg Sixt
}

January 23, 2003

\begin{abstract}
To a topological space $V$ we assign the bordism group $\mathfrak{N}_{n}^{\operatorname{def}}(V)$ of regularly defective maps $f: M \circ \longrightarrow V$ on closed $n$-dimensional manifolds $M$. These are triples $(M, \Delta, f)$ where $\Delta$ is a closed submanifold $\Delta \subset M$ and $f$ a continuous map $f: M \backslash \Delta \rightarrow V$.

We briefly review the construction of the defect complex $D V$ given by M. Rost in [17] and show that $\mathfrak{N}_{n}^{\text {def }}(V)$ is isomorphic to ordinary bordism $\mathfrak{N}_{n}(D V)$. The bordism classes in $\mathfrak{N}_{n}^{\text {def }}(V) \cong \mathfrak{N}_{n}(D V)$ are detected by characteristic numbers twisted with cohomology classes of $D V$. Some of these numbers can be described without reference to the defect complex. As an example we treat the case of the circle $V=S^{1}$. We compute $\mathfrak{N}_{n}^{\operatorname{def}}\left(S^{1}\right)$, construct a basis and a complete set of characteristic numbers.
\end{abstract}

\section{Introduction}

By a regularly defective map we mean a triple $(M, \Delta, f)$ consisting of a compact manifold $M$, a closed submanifold $\Delta \subset M$ and a continuous map $f: M \backslash \Delta \rightarrow V$ into a topological space $V$. We additionally require that $\Delta$ be transverse to the boundary $\partial M$ of $M$. Usually the defect set $\Delta$ will be suppressed in the notation and we will write $f: M \circ \longrightarrow V$.

Initially, interest in defective maps arose from the physics of ordered media, where $M$ is thought of as the coordinate space of a collection of particles, e.g. a domain in $\mathbb{R}^{3}$, cf. [11], [12] or [15]. A map $f: M \backslash \Delta \rightarrow V$ encodes some additional piece of information like the orientation of the particles. Famous examples are axial or biaxial nematics, superfluid ${ }^{3} \mathrm{He}$, see [1], [7], [10], [13]. Physicists also have considered some invariants distinguishing topologically different defective maps $f$. Probably the simplest ones are obtained 
by considering the homotopy class of the restriction of $f$ to a tubular neighbourhood of the defect set, in particular the defect indices considered in [11], [14], and [1], [2].

We consider the natural notion of bordism on such maps: Two regularly defective maps $f: M \circ \longrightarrow V$ and $f^{\prime}: M^{\prime} \circ \longrightarrow V$ with defect sets $\Delta, \Delta^{\prime}$ are bordant if there is a regulary defective map $F: W \circ \longrightarrow$ with defect set $\Gamma$ such that

1. $\partial W=M \dot{ن} M^{\prime}$,

2. $\partial \Gamma=\Delta \dot{\cup} \Delta^{\prime}$ and

3. $\left.F\right|_{M}=f,\left.F\right|_{M^{\prime}}=f^{\prime}$.

Taking disjoint union defines an addition on the set of equivalence classes. We obtain the bordism group of regularly defective maps on $n$-dimensional manifolds which we denote by $\mathfrak{N}_{n}^{\text {def }}(V)$. If $V=*$ consists of a point only we get bordism of pairs, which will be considered below.

Let $f: M \circ \longrightarrow V$ be a regularly defective map with defect set $\Delta$ and fix a component $\Delta_{0} \subset \Delta$. Consider the restriction of $f$ to the sphere bundle $S N$ of the normal bundle $N$ of $\Delta$. Choosing a fibre $S N_{x}$ of $\left.S N\right|_{\Delta_{0}}, x \in \Delta_{0}$, and a homeomorphism $h: S^{k} \cong S N_{x}$ we get a map $f \circ h: S^{k} \rightarrow V$. On the set $\left[S^{k}, V\right]$ of homotopy classes of maps $S^{k} \rightarrow V$ we have an involution \pm induced by reversing the orientation of $S^{k}$. The local defect index of $f$ at the component $\Delta_{0}$ is the class $\iota\left(f, \Delta_{0}\right)=[f \circ h] \in\left[S^{k}, V\right] / \pm$. It does not depend on the choice of $x$ and $h$. Up to sign it is the primary obstruction to extending $f$ over all of $\Delta_{0}$, cf. [3]. Regularly defective maps with $\iota\left(f, \Delta_{0}\right) \neq 0$ for each component $\Delta_{0}$ of the defect set are called topologically stable in the physics literature. In this case the defect can not be diminished by deformation, i.e. $f$ is not homotopic to a map extending to a superset of $M \backslash \Delta$.

We will also include the local defect index in the bordism groups. For a prescribed subset $\Lambda \subset \bigcup_{k}\left[S^{k}, V\right] / \pm$, a $\Lambda$-defective map $f: M \circ \longrightarrow V$ is a regularly defective map all of whose local defect indices are contained in $\Lambda$. Requiring the maps $F, f^{\prime}$ and $f$ in the above definition to be $\Lambda$-defective leads to the bordism groups $\mathfrak{N}_{n}^{\text {def }, \Lambda}(V)$.

In [17] M. Rost constructs the representing space $D_{\Lambda} V$ for the set $D_{\Lambda}(M, V)$ of concordance classes of $\Lambda$-defective maps $M \circ \longrightarrow V$ by suitably enlarging $V$ such that each $\Lambda$-defective map $f: M \circ \longrightarrow V$ induces a continuous map $F: M \rightarrow D_{\Lambda} V$, cf. section 2. He obtains a bijection $D_{\Lambda}(M, V) \rightarrow\left[M, D_{\Lambda} V\right]$. We do not need this result here but rely on 
the corresponding statement for bordism. Along the lines of [17] we obtain in section 2 a natural identification $\mathfrak{N}_{n}^{\text {def, } \Lambda}(V)=\mathfrak{N}_{n}\left(D_{\Lambda} V\right)$. Since $\mathfrak{N}_{n}\left(D_{\Lambda} V\right) \cong \bigoplus_{j=0}^{n} \mathfrak{N}_{j}(*) \otimes H_{n-j}\left(D_{\Lambda} V, \mathbb{Z}_{2}\right)$, cf. [4], the $\Lambda$-defective bordism groups can then be computed from the $\mathbb{Z}_{2}$-homology of the defect complex.

The bordism class of a regularly defective map $f: M \circ \longrightarrow V$ is determined by the characteristic numbers

$$
\left\langle w_{I}(M) \smile F^{*} \alpha,[M]\right\rangle
$$

where $\alpha \in H^{*}\left(D_{\Lambda} V\right)$ and $F: M \rightarrow D_{\Lambda} V$ extends $f$. In section 3 we describe some of these geometrically, i.e. without reference to the defect complex. For fixed $\lambda \in \Lambda$ we denote by $\Delta^{(\lambda)}$ the union of those components of $\Delta$ with local defect index $\lambda$ and by $N^{(\lambda)}$ and $S N^{(\lambda)}$ the corresponding bundles over $\Delta^{(\lambda)}$.

We consider two types of characteristic numbers for regularly defective maps. First, omitting the map $f$ defines for each $\lambda \in \Lambda$ a natural map $\mathfrak{N}_{n}^{\text {def }, \Lambda}(V) \rightarrow \mathfrak{N}_{n}^{\text {def }}(*)=\mathfrak{N}_{n}^{\text {pair }},[M, \Delta, f: M \backslash \Delta \rightarrow V] \mapsto\left[M, \Delta^{(\lambda)}\right]$ to bordism of pairs. By Theorem 1 in [19] this is completely described by the StiefelWhitney numbers $\left\langle w_{I}(T M),[M]\right\rangle$ of $M$ and the characteristic numbers

$$
\mathfrak{Y}_{\lambda, I, J}(f)=\left\langle w_{I}(\Delta) \smile w_{J}(N),\left[\Delta^{(\lambda)}\right]\right\rangle .
$$

Second we can restrict the map $f$ to the sphere bundle $\pi: S N \rightarrow \Delta$ of the normal bundle of the defect set. From the splitting $T S N^{(\lambda)}=\pi^{*} T \Delta^{(\lambda)} \oplus$ $T_{F} S N^{(\lambda)}$ we construct the characteristic numbers

$$
\mathfrak{Z}_{\lambda, \alpha, I, J}(f)=\left\langle w_{I}(\Delta) \smile w_{J}(N) \smile f^{*} \alpha,\left[S N^{(\lambda)}\right]\right\rangle
$$

for $\alpha \in H^{*}(V)$.

Section 4 deals with regularly defective bordism of the circle $V=S^{1}$. In Theorem 4.1.1 we calculate the (co)homology of $D_{\Lambda}\left(S^{1}\right)$ and thereby $\mathfrak{N}_{*}^{\text {def, } \Lambda}\left(S^{1}\right)$. A basis for $\mathfrak{N}_{*}^{\text {def, } \Lambda}\left(S^{1}\right)$ is given in section 4.2. For $V=S^{1}$ the $\mathfrak{Z}_{\lambda, \alpha, I, J}(f)$ are determined by the $\mathfrak{Y}_{\lambda, I, J}(f)$. Nonetheless we obtain a complete set of geometrically defined characteristic numbers for $\mathfrak{N}_{*}^{\text {def, } \Lambda}\left(S^{1}\right)$, $\Lambda \subset \mathbb{Z} \backslash 0$ in Theorem 4.3.1.

The bordism groups of normal coverings with Galois group $\mathbb{Z}$ are $\mathfrak{N}_{*}\left(S^{1}\right)$. Analogously $\mathfrak{N}_{*}^{\text {def }}\left(S^{1}\right)$ may be identified with cobordism of regularly branched $\mathbb{Z}$-coverings. These are branched coverings $X \rightarrow M$ in the sense of [6] with the following additional properties: First they are required to have a submanifold 
$\Delta$ of $M$ as branching, or "singular" set. Second they are to carry an action of the integers $\mathbb{Z}$ on $X$ which is transitive and free on the fibres over $M \backslash \Delta$. It is shown in [6] that, via completion, branched coverings $g: X \rightarrow M$ with singular set $\Delta$ biuniquely correspond to unbranched coverings over $M \backslash \Delta$. Taking the classifying map $f: M \backslash \Delta \rightarrow S^{1}=B \mathbb{Z}$ relates these to defective maps to $S^{1}$.

A similiar calculation is performed in [16] for $V=\mathbb{R} \mathbb{P}^{\infty}$, thus producing a branched analogue to the computation of line field cobordism by Koschorke, [9]. It turns out that $\left[f: M \circ \longrightarrow \mathbb{R P}^{\infty}\right] \in \mathfrak{N}_{*}^{\text {def }, \Lambda}\left(\mathbb{R P}^{\infty}\right)$ is determined by the bordism class of $M$ and the $\mathfrak{Z}_{\lambda, \alpha, I, J}(f)$.

Finally in section 5 we compute the invariants $\mathfrak{Z}_{\lambda, \alpha, I, J}(f)$ for some examples showing that in general they give information neither contained in the local defect index nor in the characteristic numbers of bordism of pairs.

We are grateful to Prof. K. Jänich for inspiring this research and to the referee for many valuable hints and suggestions.

\section{The Defect Complex}

We review the construction in [17] of the defect complex $D_{\Lambda} V$ of a topological space $V$. Let $\Lambda \subset \bigcup_{k=1}^{\infty}\left[S^{k}, V\right] / \pm$ which we sometimes view as a $\mathbb{Z}_{2}$-invariant subset $\Lambda \subset \bigcup_{k=1}^{\infty}\left[S^{k}, V\right]$. Let $E O(k) \rightarrow B O(k)$ denote the universal $O(k)$ bundle and $\gamma^{k}=E O(k) \times_{O(k)} \mathbb{R}^{k}$ the universal vector bundle. We endow the set $C\left(S^{k-1}, V\right)$ of continuous maps $S^{k-1} \rightarrow V$ with the compact-open topology and the $O(k)$-action $(g, f) \mapsto f \circ g^{-1}$ for $g \in O(k)$ and $f \in C\left(S^{k-1}, V\right)$.

Let $C_{\Lambda}\left(S^{k-1}, V\right)$ be the subspace of maps with homotopy class in $\Lambda$. For a $k$-dimensional $\mathbb{R}$-vector bundle $N \rightarrow \Delta$ we consider the associated $C_{\Lambda}\left(S^{k-1}, V\right)$-bundle

$$
C_{\Lambda}(S N, V)=\bigcup_{x \in \Delta} C_{\Lambda}\left(S N_{x}, V\right)=P_{O(k)}(N) \times_{O(k)} C_{\Lambda}\left(S^{k-1}, V\right) \rightarrow \Delta,
$$

where $P_{O(k)}(N) \rightarrow \Delta$ is the orthonormal frame bundle of $N$. Let

$$
\Delta_{\Lambda}^{k}=C_{\Lambda}\left(S \gamma^{k}, V\right)=E O(k) \times_{O(k)} C_{\Lambda}\left(S^{k-1}, V\right) \stackrel{\pi_{\Lambda}^{k}}{\longrightarrow} B O(k)
$$

denote the classifying $C_{\Lambda}\left(S^{k-1}, V\right)$-bundle. Then $C_{\Lambda}(S N, V)=\nu^{*} \Delta_{\Lambda}^{k}$ for a classifying map $\nu: \Delta \rightarrow B O(k)$. 
Denote by $E_{\Lambda}^{k}=\left(\pi_{\Lambda}^{k}\right)^{*} \gamma^{k}$ the pull-back of $\gamma^{k}$ to $\Delta_{\Lambda}^{k}$ and let $D E_{\Lambda}^{k}, S E_{\Lambda}^{k}$ denote its disc respectively sphere bundle. The fibre of $S E_{\Lambda}^{k}$ over a point $q \in$ $B O(k)$ is canonically $\left(S \gamma_{\Lambda}^{k}\right)_{q} \times C_{\Lambda}\left(\left(S \gamma_{\Lambda}^{k}\right)_{q}, V\right)$. Hence we have the evaluation map $a_{\Lambda}^{k}: S E_{\Lambda}^{k} \rightarrow V$. We let $\Delta_{\Lambda}, E_{\Lambda}, D E_{\Lambda}, S E_{\Lambda}, a_{\Lambda}$ denote the union over all $k \geq 1$ of the corresponding objects and use $a_{\Lambda}$ to glue

$$
D_{\Lambda} V:=D E_{\Lambda} \cup_{a_{\Lambda}} V \text {. }
$$

This set $D_{\Lambda} V$ is called the $\Lambda$-defect complex and $\Delta_{\Lambda}$ the universal defect set.

Two $\Lambda$-defective maps $f_{i}: M \circ \longrightarrow V, i=0,1$ are concordant if there is a $\Lambda$ defective map $\tilde{f}: M \times[0,1] \circ \longrightarrow V$ extending $f_{i}: M \times\{i\} \circ \longrightarrow V$. If $F: M \rightarrow D_{\Lambda} V$ is transverse to the universal defect set $\Delta$ (i.e. the induced section of $F^{*} E_{\Lambda}^{\prime}$ is transverse to the zero section, $E_{\Lambda}^{\prime}$ the pull-back of $E_{\Lambda}$ over itself), then $\Delta:=F^{-1}\left(\Delta_{\Lambda}\right)$ is a submanifold of $M$. Viewing $\Delta_{\Lambda} \subset \stackrel{\circ}{D} E_{\Lambda} \subset D_{\Lambda} V$ as the 0section we may define $R$ to be the obvious retraction $D_{\Lambda} V \backslash \Delta_{\Lambda} \rightarrow V$. Then, $R \circ F: M \circ \longrightarrow V$ is a $\Lambda$-defective map with defect set $\Delta$. It is shown in [17] that this construction induces a bijection $\mathfrak{R}:\left[M, D_{\Lambda} V\right] \stackrel{[F] \mapsto[R \circ F]}{\longrightarrow} D_{\Lambda}(M, V)$ of the set of homotopy classes of maps $M \rightarrow D_{\Lambda} V$ with the set $D_{\Lambda}(M, V)$ of concordance classes of $\Lambda$-defective maps $M \circ \longrightarrow V$.

We rely on the following immediate consequence of this construction.

Proposition 2.1 For each $n$ there is a canonical isomorphism

$$
\begin{aligned}
\mathfrak{N}_{n}\left(D_{\Lambda} V\right) & \cong \mathfrak{N}_{n}^{\text {def }, \Lambda}(V) \\
{[F] } & \longmapsto[R \circ F],
\end{aligned}
$$

where we have chosen a representative $F$ transverse to the universal defect set $\Delta_{\Lambda}$.

Proof: In [17], the inverse map $\mathfrak{L}: D_{\Lambda}(M, V) \rightarrow\left[M, D_{\Lambda} V\right]$ of $\mathfrak{R}$ is obtained by linear extension as follows. Let $f: M \backslash \Delta \rightarrow V$ be a $\Lambda$-defective map and $\nu: \Delta \rightarrow B O(k), \hat{\nu}: N \rightarrow \gamma^{k}$ be a classifying map for the normal bundle $N$ of $\Delta$. The map $f$ defines a section of the bundle $C(S N, V)=\nu^{*} \Delta_{\Lambda}^{k}$ defined above. Therefore we have a unique lift $\psi: \Delta \rightarrow \Delta_{\Lambda}^{k}, \hat{\psi}: N \rightarrow E_{\Lambda}^{k}$ of maps of vector bundles such that $\left.f\right|_{S N}=\left.a_{\Lambda}^{k} \circ \hat{\psi}\right|_{S N}$. Glueing $\left.f\right|_{M \backslash D N}$ with $\left.\hat{\psi}\right|_{D N}$ along $S N$ yields a map $L(f): M \rightarrow D_{\Lambda} V$. This map represents $\mathfrak{L}([f])$ and will be called a linear extension of $f$ in the sequel.

Applying $R \circ-$ resp. $L(-)$ to bordisms one easily sees that $\mathfrak{R}$ and $\mathfrak{L}$ induce well defined maps $\mathfrak{R}^{\prime}: \mathfrak{N}_{n}\left(D_{\Lambda} V\right) \rightarrow \mathfrak{N}_{n}^{\text {def, } \Lambda}(V),[F] \mapsto[R \circ F]$ and $\mathfrak{L}^{\prime}: \mathfrak{N}_{n}^{\text {def, },}(V) \rightarrow \mathfrak{N}_{n}\left(D_{\Lambda} V\right)$. 
Since $\mathfrak{R} \circ \mathfrak{L}=$ id and $\mathfrak{L} \circ \mathfrak{R}=$ id we obviously get $\mathfrak{R}^{\prime} \circ \mathfrak{L}^{\prime}=$ id and $\mathfrak{L}^{\prime} \circ \mathfrak{R}^{\prime}=\mathrm{id}$.

\section{Characteristic numbers for $\mathfrak{N}_{n}^{\text {def, } \Lambda}(V)$}

The bordism class of $f: M \backslash \Delta \rightarrow V$ is determined by the characteristic numbers $\left\langle w_{I}(M) \smile F^{*} \alpha,[M]\right\rangle$, where $F$ is a linear extension of $f$ as defined in the proof of proposition 2.1. In the following, we will investigate the relation between these numbers and the invariants $\mathfrak{Y}_{\lambda, I, J}(f)$ and $\mathfrak{Z}_{\lambda, \alpha, I, J}(f)$. For $q \geq 0$ and $\lambda \in \Lambda \cap\left[S^{k-1}, V\right]$ we define $\kappa_{\lambda}^{q}: H^{q}\left(\Delta_{\bar{\lambda}}\right) \rightarrow H^{q+k}\left(D_{\Lambda} V\right)$ as the composition

$$
\begin{aligned}
H^{q}\left(\Delta_{\bar{\lambda}}\right) \stackrel{\Phi_{\bar{\lambda}}}{\cong} H^{q+k}\left(D E_{\bar{\lambda}}, S E_{\bar{\lambda}}\right) & \stackrel{\left(\iota_{\bar{\lambda}}^{*}\right)^{-1}}{\cong} H^{q+k}\left(D_{\Lambda} V, D_{\Lambda} V \backslash \stackrel{\circ}{D} E_{\bar{\lambda}}\right) \stackrel{J_{\bar{\lambda}}^{*}}{\longrightarrow} \\
& \stackrel{J_{\bar{\lambda}}^{*}}{\longrightarrow} H^{q+k}\left(D_{\Lambda} V\right),
\end{aligned}
$$

where $\Phi_{\bar{\lambda}}$ is the Thom isomorphism, $\iota_{\bar{\lambda}}:\left(D E_{\bar{\lambda}}, S E_{\bar{\lambda}}\right) \rightarrow\left(D_{\Lambda} V, D_{\Lambda} V \backslash \stackrel{\circ}{D} E_{\bar{\lambda}}\right)$ the canonical map and $\jmath_{\bar{\lambda}}:\left(D_{\Lambda} V, \emptyset\right) \rightarrow\left(D_{\Lambda} V, D_{\Lambda} V \backslash D_{\bar{\lambda}}\right)$ the inclusion.

Additionally, we define $\mu_{\lambda}^{q}: H^{q}\left(S E_{\bar{\lambda}}\right) \rightarrow H^{q+1}\left(D_{\Lambda} V\right)$ as the composition

$$
\begin{aligned}
H^{q}\left(S E_{\bar{\lambda}}\right) \stackrel{\delta}{\longrightarrow} H^{q+1}\left(D E_{\bar{\lambda}}, S E_{\bar{\lambda}}\right) \stackrel{\left(\iota_{\bar{\lambda}}^{*}\right)^{-1}}{\longrightarrow} H^{q+1}\left(D_{\Lambda} V, D_{\Lambda} V \backslash \stackrel{\circ}{D} E_{\bar{\lambda}}\right) \stackrel{J_{\bar{\lambda}}^{*}}{\longrightarrow} \\
\stackrel{J_{\bar{\lambda}}^{*}}{\longrightarrow} H^{q+1}\left(D_{\Lambda} V\right) .
\end{aligned}
$$

Proposition 3.2 Then we have

$$
\mathfrak{Y}_{\lambda, I, J}(f)=\left\langle w_{I}(M) \smile F^{*} \kappa_{\lambda}^{*}\left(w_{J}\left(E_{\bar{\lambda}}\right)\right),[M]\right\rangle .
$$

Proof: Let $\Phi: H^{q}\left(\Delta^{(\lambda)}\right) \cong H^{q+k}\left(D N^{(\lambda)}, S N^{(\lambda)}\right)$ be the Thom isomorphism and $\iota:\left(D N^{(\lambda)}, S N^{(\lambda)}\right) \hookrightarrow\left(M, M \backslash \stackrel{\circ}{D} N^{(\lambda)}\right), \jmath:(M, \emptyset) \hookrightarrow\left(M, M \backslash \stackrel{\circ}{D} N^{(\lambda)}\right)$ the inclusions. Then we have

$$
\begin{aligned}
\mathfrak{Y}_{\lambda, I, J}(f) & =\left\langle w_{I}\left(\left.T M\right|_{\Delta^{(\lambda)}}\right) \smile w_{J}\left(N^{(\lambda)}\right),\left[\Delta^{(\lambda)}\right]\right\rangle \\
& =\left\langle w_{I}\left(\left.T M\right|_{D N^{(\lambda)}}\right) \smile \Phi\left(w_{J}\left(N^{(\lambda)}\right)\right),\left[D N^{(\lambda)}, S N^{(\lambda)}\right]\right\rangle \\
& =\left\langle w_{I}(M) \smile\left(\iota^{*}\right)^{-1} \Phi\left(w_{J}\left(N^{(\lambda)}\right)\right), \iota_{*}\left[D N^{(\lambda)}, S N^{(\lambda)}\right]\right\rangle \\
& =\left\langle w_{I}(M) \smile \jmath^{*}\left(\iota^{*}\right)^{-1} \Phi\left(\left.F\right|_{\Delta^{(\lambda)}}\right)^{*} w_{J}\left(E_{\bar{\lambda}}\right),[M]\right\rangle .
\end{aligned}
$$

Since $\jmath^{*}\left(\iota^{*}\right)^{-1} \Phi\left(\left.F\right|_{\Delta(\lambda)}\right)^{*}=F^{*} \kappa_{\lambda}^{*}$ the proposition is proved. 
Proposition 3.3 Let $\bar{\pi}_{\bar{\lambda}}: S E_{\bar{\lambda}} \rightarrow \Delta_{\bar{\lambda}}$ denote the projection. Then we have

$$
\mathfrak{Z}_{\lambda, \alpha, I, J}(f)=\left\langle w_{I}(M) \smile F^{*} \mu_{\lambda}^{*}\left(\bar{\pi}_{\bar{\lambda}}^{*} w_{J}\left(E_{\bar{\lambda}}\right) \smile a_{\bar{\lambda}}^{*} \alpha\right),[M]\right\rangle .
$$

Proof: Let $\iota_{S N^{(\lambda)}}: S N^{(\lambda)} \hookrightarrow D N^{(\lambda)}, \iota:\left(D N^{(\lambda)}, S N^{(\lambda)}\right) \hookrightarrow\left(M, M \backslash \stackrel{\circ}{D} N^{(\lambda)}\right)$ and $\jmath:(M, \emptyset) \hookrightarrow\left(M, M \backslash \stackrel{\circ}{D} N^{(\lambda)}\right)$ denote the inclusions. Then we have

$$
\begin{aligned}
& \mathfrak{Z}_{\lambda, \alpha, I, J}(f)=\left\langle\left(\iota_{S N^{(\lambda)}}\right)^{*} w_{I}\left(D N^{(\lambda)}\right) \smile\left(\pi_{S N^{(\lambda)}}\right)^{*} w_{J}\left(N^{(\lambda)}\right) \smile\left(\left.f\right|_{S N^{(\lambda)}}\right)^{*} \alpha,\left[S N^{(\lambda)}\right]\right\rangle \\
& \quad=\left\langle w_{I}\left(D N^{(\lambda)}\right) \smile \delta\left(\left(\pi_{S N^{(\lambda)}}\right)^{*} w_{J}\left(N^{(\lambda)}\right) \smile\left(\left.f\right|_{S N^{(\lambda)}}\right)^{*} \alpha\right),\left[D N^{(\lambda)}, S N^{(\lambda)}\right]\right\rangle \\
& \quad=\left\langle w_{I}(M) \smile\left(\iota^{*}\right)^{-1} \delta\left(\left(\pi_{S N^{(\lambda)}}\right)^{*} w_{J}\left(N^{(\lambda)}\right) \smile\left(\left.f\right|_{S N^{(\lambda)}}\right)^{*} \alpha\right), \iota_{*}\left[D N^{(\lambda)}, S N^{(\lambda)}\right]\right\rangle \\
& \quad=\left\langle w_{I}(M) \smile J^{*}\left(\iota^{*}\right)^{-1} \delta\left(\left(\pi_{S N^{(\lambda)}}\right)^{*} w_{J}\left(N^{(\lambda)}\right) \smile\left(\left.f\right|_{S N^{(\lambda)}}\right)^{*} \alpha\right),[M]\right\rangle .
\end{aligned}
$$

Let $\xi: N \rightarrow E_{\bar{\lambda}}$ denote the isometric bundle map, equal to $F$ on $D N$. Then

$$
\left(\pi_{S N^{(\lambda)}}\right)^{*} w_{J}\left(N^{(\lambda)}\right) \smile\left(\left.f\right|_{S N^{(\lambda)}}\right)^{*} \alpha=\left(\left.\xi\right|_{S N^{(\lambda)}}\right)^{*}\left(\bar{\pi}_{\bar{\lambda}}^{*} w_{J}\left(E_{\bar{\lambda}}\right) \smile a_{\bar{\lambda}}^{*} \alpha\right)
$$

and using $\jmath^{*}\left(\iota^{*}\right)^{-1} \delta\left(\left.\xi\right|_{S N^{(\lambda)}}\right)^{*}=F^{*} \mu_{\lambda}^{*}$ we have proved proposition 3.3.

\section{Regularly defective bordism of the circle}

\subsection{Homology of $D_{\Lambda}\left(S^{1}\right)$}

In the sequel (co)homology is always understood with $\mathbb{Z}_{2}$-coefficients. In this section we think of the set $\Lambda$ of admitted defect indices as a symmetric subset $\Lambda \subset \pi_{1}\left(S^{1}\right)=\mathbb{Z}, \Lambda=\Lambda_{+} \cup-\Lambda_{+}$with $\Lambda_{+} \subset \mathbb{N}_{0}$

Theorem 4.1.1 Let $\Lambda_{+}^{e v}=\Lambda_{+} \cap 2 \mathbb{Z}, \phi: \bigoplus_{\lambda \in \Lambda_{+}} \mathbb{Z}_{2} \rightarrow \mathbb{Z}_{2},\left(a_{\lambda}\right)_{\lambda} \mapsto \sum_{\lambda} \lambda a_{\lambda}$ and assume $0 \neq \Lambda$. Then

$$
H_{k}\left(D_{\Lambda} S^{1}\right) \cong \begin{cases}\mathbb{Z}_{2} & \text { for } k=0 \\ \mathbb{Z}_{2} / \operatorname{im}(\phi) & \text { for } k=1 \\ \operatorname{ker}(\phi) \subset \bigoplus_{\lambda \in \Lambda_{+}} \mathbb{Z}_{2} & \text { for } k=2 \\ \bigoplus_{\lambda \in \Lambda_{+}^{\text {ev }}} \mathbb{Z}_{2} & \text { for } k \geq 3\end{cases}
$$

and

$$
H^{k}\left(D_{\Lambda} S^{1}\right)=\operatorname{Hom}\left(H_{k}\left(D_{\Lambda} S^{1}\right), \mathbb{Z}_{2}\right) \cong \begin{cases}\mathbb{Z}_{2} & \text { for } k=0 \\ \operatorname{ker}(\psi) & \text { for } k=1 \\ \left(\prod_{\lambda \in \Lambda_{+}} \mathbb{Z}_{2}\right) / \operatorname{im}(\psi) & \text { for } k=2 \\ \prod_{\lambda \in \Lambda_{+}^{e v}} \mathbb{Z}_{2} & \text { for } k \geq 3\end{cases}
$$


where $\psi: \mathbb{Z}_{2} \rightarrow \prod_{\lambda \in \Lambda_{+}} \mathbb{Z}_{2}, 1 \mapsto(\lambda \bmod 2)_{\lambda \in \Lambda_{+}}$. If $0 \in \Lambda$ then $H_{k}\left(D_{\Lambda} S^{1}\right) \cong$ $H_{k}\left(D_{\Lambda \backslash 0} S^{1}\right) \oplus \mathbb{Z}_{2}^{k-1}$ and $H^{k}\left(D_{\Lambda} S^{1}\right) \cong H^{k}\left(D_{\Lambda \backslash 0} S^{1}\right) \times \mathbb{Z}_{2}^{k-1}$ (reading $\mathbb{Z}_{2}^{0}=$ $\left.\mathbb{Z}_{2}^{-1}=0\right)$.

For the proof of the theorem consider the subspaces

$$
\begin{aligned}
C_{\Lambda}\left(S^{1}, S^{1}\right) & :=\left\{f: S^{1} \rightarrow S^{1} \mid \operatorname{deg}(f) \in \Lambda\right\}, \\
C_{\Lambda}^{\text {nor }}\left(S^{1}, S^{1}\right) & :=\left\{f: S^{1} \rightarrow S^{1} \mid \underset{\lambda \in \Lambda}{\exists} \underset{z_{0} \in S^{1}}{\exists} \underset{z \in S^{1}}{\forall} f(z)=z_{0} z^{\lambda}\right\}
\end{aligned}
$$

of $C\left(S^{1}, S^{1}\right)$. Let $\lambda>0$ and let $\bar{\lambda}:=\{\lambda,-\lambda\}$. Obviously, $C_{\lambda}^{\text {nor }}\left(S^{1}, S^{1}\right)$ is a strong deformation retract of $C_{\lambda}\left(S^{1}, S^{1}\right)$. The deformation of the identity into a retraction can be chosen to be compatible with the $S O(2)$-action on $C_{\lambda}\left(S^{1}, S^{1}\right)$. Therefore, $\Delta_{\lambda}^{\text {nor }}:=E O(2) \times_{S O(2)} C_{\lambda}^{\text {nor }}\left(S^{1}, S^{1}\right)$ is a strong deformation retract of

$$
\Delta_{\bar{\lambda}}=E O(2) \times_{O(2)} C_{\bar{\lambda}}\left(S^{1}, S^{1}\right)=E O(2) \times_{S O(2)} C_{\lambda}\left(S^{1}, S^{1}\right) .
$$

We identify $S O(2)=S^{1}$, and consider the $S^{1}$-action $S^{1} \times S^{1} \rightarrow S^{1},(w, z) \mapsto$ $\alpha(w) z$ on $S^{1}$, where $\alpha: S^{1} \rightarrow S^{1}, w \mapsto w^{-\lambda}$. Then the homeomorphism $C_{\lambda}^{\text {nor }}\left(S^{1}, S^{1}\right) \rightarrow S^{1}, f \mapsto f(1)$ is compatible with the $S^{1}$-actions and we get

$$
\Delta_{\lambda}^{\text {nor }}=E O(2) \times_{\alpha} S^{1}=: \alpha_{*} E O(2) .
$$

Consider the vector bundle $\xi_{\lambda}:=\alpha_{*} E O(2) \times_{S^{1}} \mathbb{C} \rightarrow B S O(2)$. We have $c_{1}\left(\xi_{\lambda}\right)=-\lambda c_{1}$, where $c_{1} \in H^{2}(B S O(2), \mathbb{Z})$ denotes the universal first Chern class. Reducing modulo 2 we get $w_{2}\left(\xi_{\lambda}\right)=\lambda w_{2}$. Since $H^{*}(B S O(2))=\mathbb{Z}_{2}\left[w_{2}\right]$ and thus $H^{n}(B S O(2))=0$ for $n$ odd, the Gysin sequence of $p_{\lambda}: \Delta_{\lambda}^{\text {nor }}=$ $S \xi_{\lambda} \rightarrow B S O(2)$ yields an exact sequence

$0 \rightarrow H^{n-1}\left(\Delta_{\lambda}^{\text {nor }}\right) \stackrel{\phi_{n-1}}{\longrightarrow} H^{n-2}(B S O(2)) \stackrel{-\lambda w_{2}}{\longrightarrow} H^{n}(B S O(2)) \stackrel{p_{\lambda}^{*}}{\longrightarrow} H^{n}\left(\Delta_{\lambda}^{\text {nor }}\right) \rightarrow 0$

for each even $n \geq 2$. If $\lambda$ is odd, then $\smile \lambda w_{2}: H^{n-2}(B S O(2)) \rightarrow H^{n}(B S O(2))$ is an isomorphism and consequently $H^{k}\left(\Delta_{\lambda}^{\text {nor }}\right)=0$ for all $k \geq 1$.

If $\lambda$ is even, then $\smile \lambda w_{2}: H^{0}(B S O(2)) \rightarrow H^{2}(B S O(2))$ is zero and it follows that there exists a class $\alpha \in H^{1}\left(\Delta_{\lambda}^{\text {nor }}\right)$ with $\phi_{1}(\alpha) \neq 0$. Then $\delta(\alpha) \in H^{2}\left(D \xi_{\lambda}, S \xi_{\lambda}\right)$ is the Thom class of the vector bundle $\xi_{\lambda} \rightarrow B S O(2)$. Therefore the restriction of $\alpha$ to each fibre generates the first $\mathbb{Z}_{2}$-cohomology of the fibre. Recalling that $\Delta_{\bar{\lambda}} \simeq \Delta_{\lambda}^{\text {nor }}$ we obtain from the Leray-Hirsch Theorem: 
Proposition 4.1.2 Let $\lambda>0$. If $\lambda$ is odd then $H^{k}\left(\Delta_{\bar{\lambda}}\right)=0$ for $k \geq 1$. If $\lambda$ is even then there is a nontrivial class $\alpha \in H^{1}\left(\Delta_{\bar{\lambda}}\right)$ and $H^{*}\left(\Delta_{\bar{\lambda}}\right)$ is a free module over $H^{*}(B S O(2))$ with basis $\{1, \alpha\}$.

Applying the Thom isomorphism theorem, we get

Proposition 4.1.3 Let $\Lambda \subset \mathbb{Z} \backslash 0$ be a $\mathbb{Z}_{2}$-invariant subset, $\Lambda_{+}:=\Lambda \cap \mathbb{N}$ and $\Lambda_{+}^{e v}:=\Lambda_{+} \cap 2 \mathbb{Z}$. Then

$$
H_{k}\left(D_{\Lambda} S^{1}, S^{1}\right) \cong \begin{cases}0 & \text { for } k=0,1 \\ \bigoplus_{\lambda \in \Lambda_{+}} \mathbb{Z}_{2} & \text { for } k=2 \\ \bigoplus_{\lambda \in \Lambda_{+}^{e v}} \mathbb{Z}_{2} & \text { for } k \geq 3\end{cases}
$$

and

$$
H^{k}\left(D_{\Lambda} S^{1}, S^{1}\right) \cong \begin{cases}0 & \text { for } k=0,1 \\ \prod_{\lambda \in \Lambda_{+}} \mathbb{Z}_{2} & \text { for } k=2 \\ \prod_{\lambda \in \Lambda_{+}^{e v}} \mathbb{Z}_{2} & \text { for } k \geq 3 .\end{cases}
$$

Thus, in order to prove theorem 4.1 .1 for $0 \notin \Lambda$, it remains to show

Proposition 4.1.4 The boundary

$$
\partial: H_{2}\left(D_{\Lambda} S^{1}, S^{1}\right) \cong \bigoplus_{\lambda \in \Lambda_{+}} \mathbb{Z}_{2} \rightarrow H_{1}\left(S^{1}\right) \cong \mathbb{Z}_{2}
$$

is given by $\left(a_{\lambda}\right)_{\lambda \in \Lambda_{+}} \mapsto \sum_{\lambda \in \Lambda_{+}} \lambda a_{\lambda}$ and the coboundary $\delta: H^{1}\left(S^{1}\right) \cong \mathbb{Z}_{2} \rightarrow$ $H^{2}\left(D_{\Lambda} S^{1}, S^{1}\right) \cong \prod_{\lambda \in \Lambda_{+}} \mathbb{Z}_{2}$ by $1 \mapsto(\lambda \bmod 2)_{\lambda \in \Lambda_{+}}$.

Proof: Obviously, it suffices to show that $\delta: H^{1}\left(S^{1}\right) \rightarrow H^{2}\left(D_{\bar{\lambda}} S^{1}, S^{1}\right)$ is zero if and only if $\lambda$ is even.

Let $\iota: D^{2} \rightarrow D E_{\bar{\lambda}}$ denote the inclusion of a fibre of the disc bundle $D E_{\bar{\lambda}}$ and $\jmath:\left(D E_{\bar{\lambda}}, S E_{\bar{\lambda}}\right) \rightarrow\left(D_{\bar{\lambda}} S^{1}, S^{1}\right)$ the canonical map. Then we have the commutative diagram

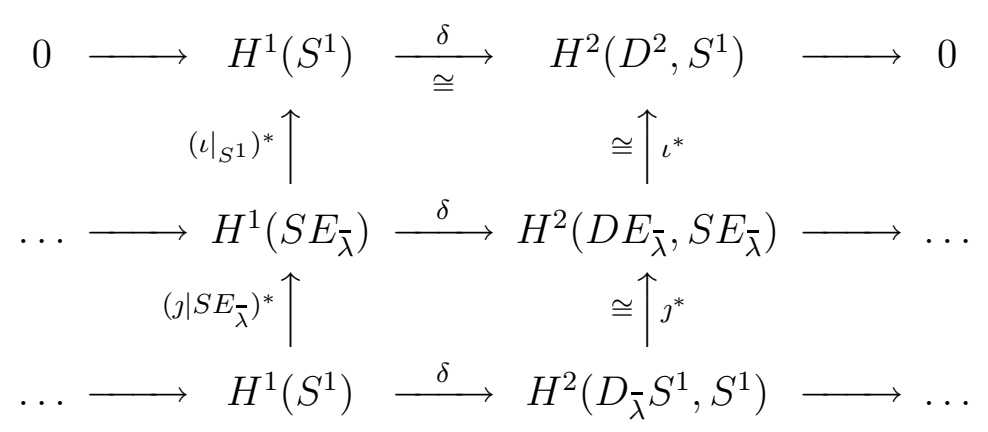


Since $\left.(\jmath \circ \iota)\right|_{S^{1}}: S^{1} \rightarrow S^{1}$ has degree $\pm \lambda$, it follows that $\left(\left.\iota\right|_{S^{1}}\right)^{*} \circ\left(\left.\jmath\right|_{S E_{\bar{\lambda}}}\right)^{*}=0$ if and only if $\lambda$ is even.

For the case $0 \in \Lambda$, observe that $\Delta_{0}^{\text {nor }}=B O(2) \times C_{0}^{\text {nor }}\left(S^{1}, S^{1}\right)$.

\subsection{A Basis for $\mathfrak{N}_{*}^{\text {def, } \Lambda}\left(S^{1}\right)$}

Let $\left\{B_{i}^{k} \mid k \geq 0, i \in I(k)\right\}$ be a set of closed differentiable manifolds with $\operatorname{dim} B_{i}^{k}=k$ such that $\left\{\left[B_{i}^{k}\right] \mid i \in I(k)\right\}$ forms a basis of $\mathfrak{N}_{k}$ for each $k \geq 0$. It is well known that one can explicitly specify such a set using products of real projective spaces and Milnor manifolds, cf. [18], [4].

Let $\left\{F_{j}^{l}: M_{j}^{l} \rightarrow X \mid l \geq 0, j \in J(l)\right\}$ be a set of singular manifolds in a topological space $X$ such that $\left\{\left(F_{j}^{l}\right)_{*}\left[M_{j}^{l}\right] \mid j \in J(l)\right\}$ is a basis of $H_{l}(X)$. Then the singular manifolds

$$
F_{j}^{l} \circ \operatorname{pr}_{2}: B_{i}^{k} \times M_{j}^{l} \rightarrow X,
$$

for $k, l \geq 0, i \in I(k), j \in J(l)$ represent a basis of $\mathfrak{N}_{k}(X)$, cf. [5]. Here $\left[M_{j}^{l}\right]$ denotes the fundamental class of $M_{j}^{l}$ over $\mathbb{Z}_{2}$.

Using this fact and the identification $\mathfrak{N}_{*}^{\text {def }, \Lambda}(V) \cong \mathfrak{N}_{*}\left(D_{\Lambda} V\right)$ of Proposition 2.1 we immediately get

Proposition 4.2.1 Let $f_{j}^{l}: M_{j}^{l} \circ \longrightarrow V, l \geq 0, j \in J(l)$ be $\Lambda$-defective maps with defect sets $\Delta_{j}^{l}$ and let $F_{j}^{l}: M_{j}^{l} \rightarrow D_{\Lambda} V$ be linear extensions. If $\left\{\left(F_{j}^{l}\right)_{*}\left[M_{j}^{l}\right] \mid j \in\right.$ $J(l)\}$ is a basis for $H_{l}\left(D_{\Lambda} V\right)$ for each $l$ then the $\Lambda$ defective maps

$$
f_{j}^{l} \circ \mathrm{pr}_{2}:\left(B_{i}^{k} \times M_{j}^{l}\right) \backslash\left(B_{i}^{k} \times \Delta_{j}^{l}\right) \rightarrow V,
$$

$k, l \geq 0, i \in I(k), j \in J(l)$ represent a basis of $\mathfrak{N}_{*}^{\text {def, } \Lambda}(V)$.

In the following we explicitly give such a set of $\Lambda$-defective maps $f_{j}^{l}, l \geq 0$, $j \in J(l)$ for the case $V=S^{1}$.

Let $\Lambda \subset \mathbb{Z} \backslash 0$ be a symmetric subset and $\Lambda_{+}:=\Lambda \cap \mathbb{N}, \Lambda_{+}^{e v}:=\Lambda_{+} \cap 2 \mathbb{Z}$. With the techniques of section 4.1 it is straightforward to show that the following $\Lambda$-defective maps fulfil the assumptions of proposition 4.2.1. For simplicity, we omit the case $0 \in \Lambda$.

Dimension 0: We take $J(0):=\{0\}, M_{0}^{0}:=\{*\}$ and choose a constant map $f_{0}^{0}: M_{0}^{0} \rightarrow S^{1}$. 
Dimension 1: If $\Lambda$ contains odd indices then $H_{1}\left(D_{\Lambda} S^{1}\right)=0$ and consequently $J(1)=\emptyset$. Else $H_{1}\left(D_{\Lambda} S^{1}\right) \cong \mathbb{Z}_{2}$ and we take $J(1):=\{1\}, M_{1}^{1}:=S^{1}$ and $f_{1}^{1}:=\mathrm{id}_{S^{1}}$.

Dimension 2: Let $\lambda_{1}<\lambda_{2}<\ldots$ be the sequence of the odd indices in $\Lambda_{+}$ and let $n \leq \infty$ be the number of such indices. Let

$$
J(2):=\Lambda_{+}^{e v} \cup\left\{\left(\lambda_{i}, \lambda_{i+1}\right) \mid 1 \leq i<n\right\} .
$$

Let $D^{2} \subset \mathbb{C}$ denote the unit disc. For each index $\lambda \in \Lambda_{+}^{e v}$ we define $g_{\lambda}: D^{2} \backslash 0 \rightarrow S^{1}, z \mapsto z^{\lambda} /\left|z^{\lambda}\right|$. Since $g_{\lambda}(z)=g_{\lambda}(-z)$, we get a well defined $\Lambda$ defective map $f_{\lambda}^{2}$ on $M_{\lambda}^{2}:=\mathbb{R P}^{2}$ by identifying antipodal points in $S^{1} \subset D^{2}$.

For $1 \leq i<n$ let $M_{\left(\lambda_{i}, \lambda_{i+1}\right)}^{2}:=S^{2}$ and define $f_{\left(\lambda_{i}, \lambda_{i+1}\right)}^{2}: S^{2} \circ S^{1}$ to be a map with $\lambda_{i+1}$ point defects of index $\lambda_{i}$ and $\lambda_{i}$ point defects of index $-\lambda_{i+1}$.

Dimension $\boldsymbol{2} \boldsymbol{k}+\mathbf{3}, \boldsymbol{k} \geq \mathbf{0}$ : Let $J(2 k+3):=\Lambda_{+}^{e v}$ and fix some $\lambda \in J(2 k+3)$. Consider the canonical bundle $\gamma_{k} \rightarrow \mathbb{C P}^{k}$. In view of the previous section we use the lens space

$$
\Delta_{\lambda}(2 k+3):=S^{2 k+1} / \lambda=S\left(\gamma_{k}^{\otimes \mathbb{C} \lambda}\right) \cong P_{S O(2)}\left(\gamma_{k}\right) \times_{S O(2)} C_{-\lambda}^{\text {nor }}\left(S^{1}, S^{1}\right)
$$

as a finite dimensional approximation of the universal defect set and $D \pi^{*} \gamma_{k} \cup_{a_{\lambda}} S^{1}$ for the defect complex. Here $\pi$ denotes the projection $S^{2 k+1} / \lambda \rightarrow \mathbb{C P}^{k}$ and $a_{\lambda}: S \pi^{*} \gamma_{k} \rightarrow S^{1}$ maps $([x], v) \mapsto z^{\lambda}$ if $v=z x, x \in S^{2 k+1}$, $v \in S^{2 k+1}, z \in S^{1}$. Let

$$
M_{\lambda}^{2 k+3}:=D \pi^{*} \gamma_{k} / \pm
$$

be obtained by identifying antipodal points in the circle bundle $S \pi^{*} \gamma_{k}$. We have a fibre bundle $M_{\lambda}^{2 k+3} \rightarrow S^{2 k+1} / \lambda$ with fibre $\mathbb{R} \mathbb{P}^{2}$. Since $\lambda$ is even there is a map $f_{\lambda, 2 k+3}: M_{\lambda}^{2 k+3} \circ S^{1}$ with defect set $S^{2 k+1} / \lambda$ and local defect index $\lambda$ induced by $a_{\lambda}$. By the discussion in the previous section the linear extension of $f_{\lambda, 2 k+3}$ maps $\left[M_{\lambda}^{2 k+3}\right]$ to the generator of $H_{2 k+3}\left(D_{\lambda} S^{1}\right)$.

Dimension $2 \boldsymbol{k}+\mathbf{2}, \boldsymbol{k} \geq \mathbf{1}$ : For $\lambda \in J(2 k+2):=\Lambda_{+}^{e v}$ let $g_{\lambda, 2 k+2}$ be the composition

$$
\Delta_{\lambda}(2 k+2):=\mathbb{R} \mathbb{P}^{2 k} \hookrightarrow \mathbb{R} \mathbb{P}^{2 k+1}=S^{2 k+1} / 2 \rightarrow S^{2 k+1} / \lambda .
$$

We define $M_{\lambda}^{2 k+2}$ to be the pull-back of $M_{\lambda}^{2 k+3}$ to $\Delta_{\lambda}(2 k+2)$ with $g_{\lambda, 2 k+2}$ and let $f_{\lambda, 2 k+2}$ be the composition $M_{\lambda}^{2 k+2} \rightarrow M_{\lambda}^{2 k+3} \circ \longrightarrow S^{1}$. Since $g_{\lambda, 2 k+2}$ induces an isomorphism in $H_{2 k}$ we get that the linear extension of $f_{\lambda, 2 k+2}$ maps $\left[M_{\lambda}^{2 k+2}\right]$ to the generator of $H_{2 k+2}\left(D_{\lambda} S^{1}\right)$. 


\subsection{Characteristic numbers for $\mathfrak{N}_{n}^{\text {def, }, \Lambda}\left(S^{1}\right)$}

Let $\Lambda \subset \mathbb{Z} \backslash 0$ be a $\mathbb{Z}_{2}$-invariant subset. In this section we prove the following:

Theorem 4.3.1 Let $f: M \circ \longrightarrow S^{1}$ be a $\Lambda$-defective map. There is a unique class $\alpha \in H^{1}(M)$ with $\left.\alpha\right|_{M \backslash \Delta}=f^{*} \varphi_{S^{1}}$, where $\varphi_{S^{1}}$ denotes the generator of $H^{1}\left(S^{1}\right)$. For each $\lambda \in \Lambda_{+}^{e v}$ there is a unique class $\beta_{\lambda} \in H^{1}\left(\Delta^{(\lambda)}\right) \subset$ $H^{1}(\Delta)$ with the following property: If $\iota: S^{1} \rightarrow \Delta^{(\lambda)}$ is any continuous map, $\hat{\iota}: S\left(\iota^{*} N^{(\lambda)}\right) \rightarrow S N^{(\lambda)}$ the canonical map over $\iota$ and $\sigma: S^{1} \rightarrow S\left(\iota^{*} N^{(\lambda)}\right)$ an arbitrary cross-section, then

$$
\left\langle\beta_{\lambda}, \iota_{*}\left[S^{1}\right]\right\rangle=\operatorname{deg}\left(\left.f\right|_{S N^{(\lambda)}} \circ \hat{\iota} \circ \sigma\right) \bmod 2 .
$$

The bordism class of $f: M \backslash \Delta \rightarrow S^{1}$ is determined by the characteristic numbers

$$
\begin{aligned}
& \left\langle w_{I}(M) \smile \alpha,[M]\right\rangle, \\
& \left\langle w_{I}\left(\left.T M\right|_{\Delta}\right) \smile w_{2}(N)^{q-1},\left[\Delta^{(\lambda)}\right]\right\rangle=\mathfrak{Y}_{\lambda, I,(0, q-1)}(f) \text { with } \lambda \in \Lambda_{+}, \\
& \left\langle w_{I}\left(\left.T M\right|_{\Delta}\right) \smile w_{2}(N)^{q-1} \smile \beta_{\lambda},\left[\Delta^{(\lambda)}\right]\right\rangle \text { with } \lambda \in \Lambda_{+}^{e v}
\end{aligned}
$$

together with the bordism class of $M$.

Let $F: M \rightarrow D_{\Lambda} S^{1}$ be a linear extension of $f$. Throughout this section let $\kappa_{\lambda}^{q}: H^{q}\left(\Delta_{\bar{\lambda}}\right) \rightarrow H^{q+2}\left(D_{\Lambda} S^{1}\right)$ denote the homomorphism (3.1) in the case $V=$ $S^{1}$. Theorem 4.3.1 is an immediate consequence of the following propositions.

First we assume that $\Lambda \subset 2 \mathbb{Z}$. Then $H^{1}\left(D_{\Lambda} V\right) \cong \mathbb{Z}_{2}$. Recall that we have $H^{1}\left(D_{\Lambda} V\right)=0$ if $\Lambda \not \subset 2 \mathbb{Z}$. Let $\eta$ be the nontrivial element in $H^{1}\left(D_{\Lambda} V\right)$.

Proposition 4.3.3 The restriction $H^{1}(M) \rightarrow H^{1}(M \backslash \Delta)$ is injective and we have $\left.\left(F^{*} \eta\right)\right|_{M \backslash \Delta}=f^{*} \varphi_{S^{1}}$, where $\varphi_{S^{1}} \in H^{1}\left(S^{1}\right)$ denotes the generator.

Proof: Since $\Delta$ has codimension 2, we have $H^{1}(M, M \backslash \Delta)=0$ and the long exact sequence yields the injectivity of the restriction. Let $j: S^{1} \rightarrow D_{\Lambda} S^{1}$ denote the inclusion. Since $H^{1}\left(D_{\Lambda} S^{1}, S^{1}\right)$ is zero, $j^{*}: H^{1}\left(D_{\Lambda} S^{1}\right) \rightarrow H^{1}\left(S^{1}\right)$ is bijective. Consequently, we have $\varphi_{S^{1}}=j^{*} \eta$. As $\left.F\right|_{M \backslash \Delta}$ is homotopic to $j \circ f$, it follows that $\left.\left(F^{*} \eta\right)\right|_{M \backslash \Delta}=f^{*} j^{*} \eta=f^{*} \varphi_{S^{1}}$.

Now, let $\Lambda$ be an arbitrary $\mathbb{Z}_{2}$-invariant subset of $\mathbb{Z} \backslash 0$. We have $H^{2}\left(D_{\Lambda} S^{1}\right) \cong$ $\left(\prod_{\lambda \in \Lambda_{+}} \mathbb{Z}_{2}\right) / \operatorname{im}(\psi)$, where $\psi$ is the homomorphism defined in theorem 4.1.1. 
Proposition 4.3.4 Let $\eta \in H^{2}\left(D_{\Lambda} S^{1}\right)$ and let $\left(a_{\lambda}\right)_{\lambda \in \Lambda_{+}} \in \prod_{\lambda \in \Lambda_{+}} \mathbb{Z}_{2}$ be an element representing $\eta$ under the isomorphism of Theorem 4.1.1. Then

$$
\left\langle w_{I}(M) \smile F^{*} \eta,[M]\right\rangle=\sum_{\lambda \in \Lambda_{+}} a_{\lambda}\left\langle w_{I}\left(\left.T M\right|_{\Delta}\right),\left[\Delta^{(\lambda)}\right]\right\rangle=\sum_{\lambda \in \Lambda_{+}} a_{\lambda} \mathfrak{Y}_{\lambda, I,(0)}(f) .
$$

Proof: Since $\Delta$ is compact, we may assume that $\Lambda$ is finite. Moreover, it suffices to consider $\lambda \in \Lambda_{+}$with $a_{\lambda}=1$ and $a_{\mu}=0$ for $\mu \in \Lambda_{+} \backslash\{\lambda\}$, hence $\eta=\kappa_{\lambda}^{0}(1)$. Proposition 3.2 yields

$$
\left\langle w_{I}(M) \smile F^{*} \eta,[M]\right\rangle=\mathfrak{Y}_{\lambda, I,(0)}(f)=\left\langle w_{I}\left(\left.T M\right|_{\Delta}\right),\left[\Delta^{(\lambda)}\right]\right\rangle .
$$

For the even dimensions $\geq 4$ we have

Proposition 4.3.5 Let $q \geq 2$ and $\eta=\left(a_{\lambda}\right)_{\lambda \in \Lambda_{+}^{e v}} \in \prod_{\lambda \in \Lambda_{+}^{e v}} \mathbb{Z}_{2} \cong H^{2 q}\left(D_{\Lambda} S^{1}\right)$. Then

$$
\begin{aligned}
\left\langle w_{I}(M) \smile F^{*} \eta,[M]\right\rangle & =\sum_{\lambda \in \Lambda_{+}^{e v}} a_{\lambda}\left\langle w_{I}\left(\left.T M\right|_{\Delta}\right) \smile w_{2}(N)^{q-1},\left[\Delta^{(\lambda)}\right]\right\rangle \\
& =\sum_{\lambda \in \Lambda_{+}^{e v}} a_{\lambda} \mathfrak{Y}_{\lambda, I,(0, q-1)}(f) .
\end{aligned}
$$

Proof: We may again assume that we have a $\lambda \in \Lambda_{+}^{e v}$ with $a_{\lambda}=1$ and $a_{\mu}=0$ for $\mu \in \Lambda_{+}^{e v} \backslash\{\lambda\}$. Then $\eta=\kappa_{\lambda}^{2 q-2}\left(w_{2}\left(E_{\bar{\lambda}}\right)^{q-1}\right)$. Proposition 3.2 yields

$$
\left\langle w_{I}(M) \smile F^{*} \eta,[M]\right\rangle=\mathfrak{Y}_{\lambda, I,(0, q-1)}(f)=\left\langle w_{I}\left(\left.T M\right|_{\Delta}\right) \smile w_{2}(N)^{q-1},\left[\Delta^{(\lambda)}\right]\right\rangle .
$$

Now, let $q \geq 1$ and $\eta=\left(a_{\lambda}\right)_{\lambda \in \Lambda_{+}^{e v}} \in \prod_{\lambda \in \Lambda_{+}^{e v}} \mathbb{Z}_{2} \cong H^{2 q+1}\left(D_{\Lambda} S^{1}\right)$. For $\lambda \in \Lambda_{+}$let $F^{(\lambda)}: \Delta^{(\lambda)} \rightarrow \Delta_{\bar{\lambda}}$ be the restriction of $F$ to $\Delta^{(\lambda)}$.

Proposition 4.3.6 For $\lambda \in \Lambda_{+}^{e v}$ let $\beta_{\lambda}$ be the generator of $H^{1}\left(\Delta_{\bar{\lambda}}\right)$ as in Proposition 4.1.2. Then

$$
\begin{aligned}
\left\langle w_{I}(M)\right. & \left.\smile F^{*} \eta,[M]\right\rangle= \\
& =\sum_{\lambda \in \Lambda_{+}^{e v}} a_{\lambda}\left\langle w_{I}\left(\left.T M\right|_{\Delta^{(\lambda)}}\right) \smile w_{2}\left(\left.N\right|_{\Delta(\lambda)}\right)^{q-1} \smile F^{(\lambda) *} \beta_{\lambda},\left[\Delta^{(\lambda)}\right]\right\rangle .
\end{aligned}
$$


Proof: Assume again that $a_{\lambda}=1$ and $a_{\mu}=0$ for $\mu \in \Lambda_{+}^{e v} \backslash\{\lambda\}$. Then we have $\eta=\kappa_{\lambda}^{2 q-1}\left(w_{2}\left(E_{\bar{\lambda}}\right)^{q-1} \smile \beta_{\lambda}\right)$. With the Thom isomorphism $\Phi$ and the inclusions $\iota:\left(D N^{(\lambda)}, S N^{(\lambda)}\right) \hookrightarrow\left(M, M \backslash \stackrel{\circ}{D} N^{(\lambda)}\right)$ and $\jmath:(M, \emptyset) \hookrightarrow(M, M \backslash$ $\left.\stackrel{\circ}{D} N^{(\lambda)}\right)$ we get

$$
\begin{aligned}
\left\langle w_{I}\left(\left.T M\right|_{\Delta(\lambda)}\right)\right. & \left.\smile w_{2}\left(N^{(\lambda)}\right)^{q-1} \smile F^{(\lambda) *} \beta_{\lambda},\left[\Delta^{(\lambda)}\right]\right\rangle \\
& =\left\langle w_{I}\left(\left.T M\right|_{D N^{(\lambda)}}\right) \smile \Phi F^{(\lambda) *}\left(w_{2}\left(E_{\bar{\lambda}}\right)^{q-1} \smile \beta_{\lambda}\right),\left[D N^{(\lambda)}, S N^{(\lambda)}\right]\right\rangle \\
& =\left\langle w_{I}(M) \smile\left(\iota^{*}\right)^{-1} \Phi F^{(\lambda) *}\left(w_{2}\left(E_{\bar{\lambda}}\right)^{q-1} \smile \beta_{\lambda}\right), \iota_{*}\left[D N^{(\lambda)}, S N^{(\lambda)}\right]\right\rangle \\
& =\left\langle w_{I}(M) \smile \jmath^{*}\left(\iota^{*}\right)^{-1} \Phi F^{(\lambda) *}\left(w_{2}\left(E_{\bar{\lambda}}\right)^{q-1} \smile \beta_{\lambda}\right),[M]\right\rangle .
\end{aligned}
$$

Using $\jmath^{*}\left(\iota^{*}\right)^{-1} \Phi F^{(\lambda) *}=F^{*} \kappa_{\lambda}^{*}$ we obtain

$$
\left\langle w_{I}\left(\left.T M\right|_{\Delta^{(\lambda)}}\right) \smile w_{2}\left(N^{(\lambda)}\right)^{q-1} \smile F^{(\lambda) *} \beta_{\lambda},\left[\Delta^{(\lambda)}\right]\right\rangle=\left\langle w_{I}(M) \smile F^{*} \eta,[M]\right\rangle .
$$

Thus, it remains to describe the classes $F^{(\lambda) *} \beta_{\lambda} \in H^{1}\left(\Delta^{(\lambda)}\right)$ for $\lambda \in \Lambda_{+}^{e v}$.

Proposition 4.3.7 Let $\lambda \in \Lambda_{+}^{e v}$, let $\iota: S^{1} \rightarrow \Delta^{(\lambda)}$ be a continuous mapping and let $\hat{\iota}: S\left(\iota^{*} N^{(\lambda)}\right) \rightarrow S N^{(\lambda)}$ denote the canonical map over $\iota$. For an arbitrary cross-section $\sigma: S^{1} \rightarrow S\left(\iota^{*} N^{(\lambda)}\right)$ we then have:

$$
\left\langle F^{(\lambda) *} \beta_{\lambda}, \iota_{*}\left[S^{1}\right]\right\rangle=\operatorname{deg}\left(\left.f\right|_{S N^{(\lambda)}} \circ \hat{\iota} \circ \sigma\right) \bmod 2 .
$$

Proof: Let $\sigma_{1}$ and $\sigma_{2}$ be two cross-sections in $S\left(\iota^{*} N^{(\lambda)}\right) \rightarrow S^{1}$. Then obviously $\operatorname{deg}\left(\left.f\right|_{S N^{(\lambda)}} \circ \hat{\iota} \circ \sigma_{2}\right)-\operatorname{deg}\left(\left.f\right|_{S N^{(\lambda)}} \circ \hat{\iota} \circ \sigma_{1}\right)$ is a multiple of $\lambda$ and consequently

$$
\operatorname{deg}\left(\left.f\right|_{S N^{(\lambda)}} \circ \hat{\iota} \circ \sigma_{2}\right) \equiv \operatorname{deg}\left(\left.f\right|_{S N^{(\lambda)}} \circ \hat{\iota} \circ \sigma_{1}\right) \bmod 2 .
$$

Therefore, is suffices to show the existence of a cross-section $\sigma$ which fulfils (4.3.8). Let $\tilde{\pi}: \Delta_{\bar{\lambda}} \rightarrow B S O(2)$ denote the projection map. As $\pi_{1}(B S O(2))=$ $0, \tilde{\pi}_{\lambda} \circ F^{(\lambda)} \circ \iota$ is null homotopic and we can assume that $\tilde{\pi}_{\lambda} \circ F^{(\lambda)} \circ \iota \equiv$ $x \in B S O(2)$. Let $\widetilde{\gamma}^{2}$ denote the universal vector bundle over $B S O(2)$. An arbitrary element $v \in S \widetilde{\gamma}_{x}^{2}$ yields a cross-section $\widetilde{\sigma}:\left.\left(\Delta_{\bar{\lambda}}\right)_{x} \rightarrow S E_{\bar{\lambda}}\right|_{\left(\Delta_{\bar{\lambda}}\right)_{x}}$. Let $\sigma: S^{1} \rightarrow S\left(\iota^{*} N^{(\lambda)}\right)$ be the cross-section induced by $\tilde{\sigma}$.

The map $a_{\bar{\lambda}} \circ \widetilde{\sigma}$ is equal to the evaluation map

$$
\left(\Delta_{\bar{\lambda}}\right)_{x}=C_{\lambda}\left(S \widetilde{\gamma}_{x}^{2}, S^{1}\right) \longrightarrow S^{1}, \quad g \longmapsto g(v)
$$


and therefore is a homotopy equivalence. Thus, $\left(a_{\bar{\lambda}} \circ \widetilde{\sigma}\right)^{*} \varphi_{S^{1}}$ is the generator of $H^{1}\left(\left(\Delta_{\bar{\lambda}}\right)_{x}\right)=\mathbb{Z}_{2}$, i.e. $\left(a_{\bar{\lambda}} \circ \tilde{\sigma}\right)^{*} \varphi_{S^{1}}=\left.\beta_{\lambda}\right|_{\left(\Delta_{\bar{\lambda}}\right)_{x}}$. We obtain

$$
\iota^{*} F^{(\lambda) *} \beta_{\lambda}=\iota^{*} F^{(\lambda) *}\left(a_{\bar{\lambda}} \circ \widetilde{\sigma}\right)^{*} \varphi_{S^{1}}=\left(\left.f\right|_{S N^{(\lambda)}} \circ \hat{\iota} \circ \sigma\right)^{*} \varphi_{S^{1}}
$$

and the proposition is proved.

Thus the characteristic numbers (4.3.2) together with the bordism class of $M$ determine all the numbers (1.1).

\section{$5 \quad$ Further Examples}

We end with some examples of nonvanishing invariants $\mathfrak{Z}_{\lambda, \alpha, I, J}(f)$ distinguishing bordism of regularly defective maps from bordism of pairs.

Example: The unit tangent bundle of $\mathbb{R P}^{2 k}$ is explicitly given as $S T \mathbb{R} \mathbb{P}^{2 k}=$ $\left\{(x, y) \in S^{2 k} \times S^{2 k} \mid x \perp y\right\} / \sim$, with the antipodal identification $(x, y) \sim$ $(-x,-y)$. For independent $x, y \in \mathbb{R}^{2 k+1}$, let $\langle x, y\rangle \in G_{2}^{+}\left(\mathbb{R}^{2 k+1}\right)$ denote the oriented subspace spanned by these vectors and define a map $\hat{f}: S T \mathbb{R} \mathbb{P}^{2 k} \rightarrow$ $V=G_{2}^{+}\left(\mathbb{R}^{2 k+1}\right)$ by $[x, y] \mapsto\langle x, y\rangle$. Mapping $[x, y] \mapsto(\langle x, y\rangle,[x])$ defines a homeomorphism of $S T \mathbb{R P}^{2 k}$ with the projective bundle of the canonical bundle over $G_{2}^{+}\left(\mathbb{R}^{2 k+1}\right)$. This is the circle bundle of a 2 -dimensional vector bundle $L$ over $G_{2}^{+}\left(\mathbb{R}^{2 k+1}\right)$ and under the above identifications, the map $\hat{f}$ extends to the bundle projection of $L$. Glueing the disc bundle $D L$ of $L$ with the obvious regularly defective extension of $\hat{f}$ to the disc bundle $D T \mathbb{R P}^{2 k}$ we obtain a regularly defective map

$$
f: M=D L \cup_{S T \mathbb{R} \mathbb{P}^{2 k}} D T \mathbb{R} \mathbb{P}^{2 k} \circ V=G_{2}^{+}\left(\mathbb{R}^{2 k+1}\right)
$$

with defect set $\mathbb{R P}^{2 k}$. If $k \geq 2$ its local defect index $\lambda$ is a generator of $\mathbb{Z}=\pi_{2 k-1}(V)$. We compute the $\mathfrak{Z}_{\lambda, \alpha, I, J}(\hat{f})$.

From the Leray-Hirsch Theorem we infer that $\hat{f}^{*}$ is injective and that $H^{*}\left(S T \mathbb{R P}^{2 k}\right)$ is a free $H^{*}(V)$-module with base $\{1, y\}$ for some $y \in H^{1}\left(S T \mathbb{R P}^{2 k}\right)$. The Gysin-sequence shows that $H^{*}\left(S T \mathbb{R} \mathbb{P}^{2 k}\right) \cong$ $\mathbb{Z}_{2}[b, y] /\left(b^{2}, y^{2 k}\right)$ as graded $\mathbb{Z}_{2}$-algebras with $\operatorname{deg}(b)=2 k$ and $y$ the generator of $H^{1}\left(S T \mathbb{R P}^{2 k}\right) \cong H^{1}\left(\mathbb{R P}^{2 k}\right)$. Hence $H^{*}(V) \cong \mathbb{Z}_{2}\left[b, y^{2}\right] /\left(b^{2}, y^{2 k}\right)$. One can now easily compute the $\mathfrak{Z}_{\lambda, \alpha, I, J}(f)$. For instance taking $\alpha=y^{2 k-2} b$, $I=(1,0, \ldots, 0)$ and $J=(0)$ we get $\mathfrak{Z}_{\lambda, \alpha, I, J}(f)=1$.

For the second set of examples we need the following. 
Lemma 5.1 For multiindices $L, I$ and $l \in \mathbb{N}$ there are universal polynomials $p_{L, I, l} \in \mathbb{Z}_{2}\left[T_{1}, \ldots T_{l}\right]$ with the following property: Let $\pi: N \rightarrow \Delta$ be an $l$ dimensional vector bundle, $U_{N}$ its Thom class and $\Phi$ the Thom isomorphism. Then $P_{L}: H^{*}(\Delta) \rightarrow H^{*}(\Delta)$ mapping

$$
x \longmapsto \sum_{I \in \mathbb{N}_{0}^{t}, t \in \mathbb{N}} p_{L, I, l}\left(w_{1}, \ldots, w_{l}\right) \operatorname{Sq}^{I}(x)
$$

fulfils $\mathrm{Sq}^{L}(\Phi(x))=\Phi\left(P_{L}(x)\right)$.

Proof: We use induction on the length of $L$. For $L \in \mathbb{N}$ we compute

$$
\operatorname{Sq}^{L}(\Phi(x))=\sum_{r+s=L} \pi^{*}\left(\operatorname{Sq}^{r}(x)\right) \underbrace{\operatorname{Sq}^{s}\left(U_{N}\right)}_{=\Phi\left(w_{s}\right)}=\Phi\left(\sum_{r+s=L} w_{s} \operatorname{Sq}^{r}(x)\right)) .
$$

The assertion follows by induction using the formulae of $\mathrm{Wu}$ and Cartan. Obviously the polynomials do not depend on the bundle $\pi: N \rightarrow \Delta$.

Proposition 5.2 Let $M$ be a compact $n$-dimensional manifold and $\Delta$ a closed $k$-dimensional connected submanifold. Let $\pi: N \rightarrow \Delta$ be the normal bundle. Let $I, \tilde{I}, J, \tilde{J}, L, \tilde{L}$ be multiindices and $y \in H^{s-n+k+1}(\Delta), y \neq 0$ with $s>n-k-1$ such that

$$
\begin{aligned}
& \text { 1. } w_{n-k}(N)=0, \\
& \text { 2. } H^{n-k}(M, \Delta)=H^{s}(M, \Delta)=0, \\
& \text { 3. }\left\langle P_{L}(1) \smile w_{I}(\Delta) \smile w_{J}(N),[\Delta]\right\rangle \neq 0, \\
& \text { 4. }\left\langle P_{\tilde{L}}(y) \smile w_{\tilde{I}}(\Delta) \smile w_{\tilde{J}}(N),[\Delta]\right\rangle \neq 0 .
\end{aligned}
$$

Then there are maps $f_{1,2}: M \backslash \Delta \rightarrow V:=K\left(\mathbb{Z}_{2}, n-k-1\right) \times K\left(\mathbb{Z}_{2}, s\right)$ with the same nontrivial local defect index $\lambda$ representing different nontrivial elements in $\mathfrak{N}_{n}^{\text {def, } \lambda}(V)$.

For an explicit example choose $M=S^{n}$ and $\Delta$ any submanifold diffeomorphic to $\mathbb{R P}^{k}$ with $k$ even, $n>2 k+1, n-k<s<n, I=(1,0, \ldots, 0)$, $J=\tilde{J}=(0, \ldots, 0), L=1, \tilde{I}=(n-s-1,0 \ldots, 0), \tilde{L}=0, y=z^{s-n+k+1}$ with $z$ the generator of $H^{*}\left(\mathbb{R P}^{k}\right)$. 
Proof: The $f_{i}$ will be distinguished by suitable $\mathfrak{Z}_{\lambda, \alpha, I, J}$. Let $u$ and $v$ be the characteristic elements of $K\left(\mathbb{Z}_{2}, n-k-1\right)$ and $K\left(\mathbb{Z}_{2}, s\right)$. Let $b \in H^{n-k-1}(S N)$ with $\rho(b)=1$ where $\rho$ is the connecting homomorphism in the Gysin sequence. Let $i: S N \rightarrow M \backslash \Delta$ be the inclusion. The long exact sequence of $(M \backslash \stackrel{\circ}{D} N, S N)$ shows that there is an $x \in H^{n-k-1}(M \backslash \Delta)$ such that $i^{*}(x)=b$. There is a unique map $f: M \backslash \Delta \rightarrow K\left(\mathbb{Z}_{2}, n-k-1\right)$ such that $f^{*}(u)=x$. Analogously there is a $\tilde{x} \in H^{s}(M \backslash \Delta)$ with $i^{*}(\tilde{x})=y b$ and a $\operatorname{map} g: M \backslash \Delta \rightarrow K\left(\mathbb{Z}_{2}, s\right)$ with $g^{*}(v)=\tilde{x}$. Define $f_{1}:=f \times$ const. and $f_{2}:=f \times g$.

Now we show that both maps have nontrivial local defect indices. Since $\delta i^{*} f^{*}(u)$ is the Thom class of $N$ we know that its restriction on any fibre of $S N$ is not zero. This shows that the local defect index of $f$ is not zero for any $p \in \Delta$.

By assumption $3, \mathfrak{Z}_{\lambda, \mathrm{Sq}^{L}(u) \times 1, I, J}\left(f_{i}\right)=\mathfrak{Z}_{\lambda, \mathrm{Sq}^{L}(u), I, J}(f) \neq 0$. Hence the $f_{i}$ are not null bordant. But on the other hand $f_{1}$ and $f_{2}$ are not bordant since $\mathfrak{Z}_{\lambda, 1 \times \mathrm{Sq}} \tilde{L}_{(v), \tilde{I}, \tilde{J}}\left(f_{1}\right)=0$ and $\mathfrak{Z}_{\lambda, 1 \times \mathrm{Sq}^{\tilde{L}}(v), \tilde{I}, \tilde{J}}\left(f_{2}\right) \neq 0$.

\section{References}

[1] Bechtluft-Sachs, S., Hien, M.: The Global Defect Index. Comm. Math. Phys. 202 (1999), no. 2, 403-409

[2] Bechtluft-Sachs, S.; Hien, M.: Sets of fibre homotopy classes and twisted order parameter spaces. Comm. Math. Phys. 211 (2000), no. 2, 407-412

[3] Bechtluft-Sachs, S.; Hien, M.: The local defect index up to finite ambiguity. Topology Appl. 119 (2002), no. 2, 113-116

[4] Bröcker, T., tom Dieck, T.: Kobordismentheorie, Lecture Notes in Mathematics, Vol. 178, Springer-Verlag, Berlin-New York 1970

[5] Conner, P. E., Floyd, E. E.: Differentiable Periodic Maps, Ergebnisse der Mathematik und ihrer Grenzgebiete, Springer-Verlag, Berlin 1964

[6] Fox, R. H.: Covering spaces with singularities. A symposium in honor of S. Lefschetz, pp 243-257, 1957

[7] Jänich, K.: Topological properties of ordinary nematics in 3-space. Acta Appl. Math. 8, 65-74 (1987) 
[8] Jänich, K., Rost, M.: Regularity of line defects in 3-dimensional media. Topology 24, No. 3, 353-360 (1985)

[9] Koschorke, U.: Concordance and bordism of line fields. Invent. Math. 24 (1974), 241-268.

[10] Leggett, S. J.: A theoretical description of the new phases of liquid ${ }^{3} \mathrm{He}$. Rev. Mod. Phys. 47, 331-414 (1975)

[11] Mermin, N. D.: The topological theory of defects in ordered media. Rev. Mod. Phys. 51, 591-648 (1979)

[12] Michel, L.: Symmetry, defects and broken symmetry. Configurations. Hidden symmetry. Rev. Mod. Phys. 52, 617-651 (1980)

[13] Mermin, N. D., Lee, D. M.: Superfluid Helium 3. Scient. Am. 235, 56-71 (1976)

[14] Nakanishi, H., Hayashi, K, Mori, H.:Topological classification of unknotted ring defects. Commun. Math. Phys. 117, 203-213 (1988)

[15] Poénaru, V., Toulouse, G.: The crossing of defects in ordered media and the topology of 3-manifolds. J. Phys. 38, 887-895 (1977)

[16] Riegel, U.: Bordism of branched line bundles, to appear in Math. Nachr.

[17] Rost, M.: Abbildungsdefekte in 4-Mannigfaltigkeiten, Regensburger Mathematische Schriften 12, Regensburg 1987

[18] Thom, R.: Quelques proptiétés globales des variétés différentiables, Comment. Math. Helv. 28, 17-86 (1954)

[19] Wall, C. T. C.: Cobordism of Pairs, Comm. Math. Helv. 35 (1961), $136-145$ 